\title{
ORIGINAL
}

\section{BROTE DE PAROTIDITIS VÍRICA EN UN COLEGIO DE BIZKAIA EN 2006}

\author{
Eduardo Millán Ortuondo (1), Itxaso González Sancristóbal (2), Leyre López Soria (3), \\ Juan Emilio Echevarría Mayo (4) Visitación de Castro Laiz (2) y Nerea Muniozguren Agirre (2) \\ (1) Servicio de Medicina Preventiva del Hospital de Cruces. Bizkaia. \\ (2) Unidad de Vigilancia Epidemiológica de Bizkaia. \\ (3) Servicio de Microbiología del Hospital de Cruces. Bizkaia. \\ (4) Centro Nacional de Microbiología. Instituto de Salud Carlos III. Majadahonda. Madrid.
}

\section{RESUMEN}

Fundamento: Recientemente se han registrado brotes de parotiditis en España y en otros países desarrollados. Los motivos barajados son la baja cobertura vacunal de las poblaciones afectadas y/o la baja efectividad de las cepas vacunales empleadas. Este trabajo describe un brote de parotiditis ocurrido en Bizkaia y valora la efectividad de las cepas vacunales y la utilidad de las pruebas diagnósticas actualmente empleadas.

Métodos: Se etiquetaron como casos aquéllos con clínica compatible y vínculo epidemiológico en el periodo de estudio (febrero-mayo-2006). Se recogieron muestras de sangre para estudio de IgM e IgG y de saliva para detección de RNA y genotipo. Se averiguó el estado vacunal y la cepa empleada mediante los registros del reparto vacunal. Se realizó un análisis univariante de los datos y se obtuvieron riesgos relativos según las cepas vacunales empleadas.

Resultados: Se detectaron 63 casos; 52 eran alumnos del mismo colegio. El 50\% tenía entre 9 y 13 años. El 88,5\% de los casos del colegio estaba correctamente vacunado. La sensibilidad de la IgM fue del 9\% y la de la PCR del 37\%. El riesgo relativo de los alumnos vacunados con una primera dosis de cepa Rubini frente a los vacunados con cepa Jeryl-Lynn fue de 3,8 (IC95\% 2,27-6,49).

Conclusiones: La elevada cobertura vacunal no impide el desarrollo de brotes en lugares con un alto grado de exposición. La IgM se muestra poco sensible para el diagnóstico de parotiditis. Parece necesario replantearse las estrategias vacunales y los métodos diagnósticos actuales.

Palabras clave: Paperas. Virus de la parotiditis. Vacunación. Brote epidémico.

\section{Correspondencia:}

Eduardo Millán Ortuondo

Departamento de Sanidad. Dirección Territorial de Bizkaia.

María Díaz de Haro, 58.

48010 Bilbo-Bilbao.

Correo electrónico: edumior@gmail.com

\section{ABSTRACT \\ Outbreak of Viral Parotiditis in a School in Bizkaia, Spain, in 2006}

Background: Outbreaks of parotiditis have recently been recorded in Spain and in other developed countries. The possible reasons currently under consideration are the low degree of immunization coverage among the populations involved and/or the low degree of effectiveness of the vaccine strains employed. This study describes one outbreak of parotiditis having occurred in Bizkaia and assesses the effectiveness of the vaccine strains and the usefulness of the diagnostic tests currently used.

Methods: Those cases having compatible clinical symptoms and an epidemiological link within the period under study (February-May 2006) were labeled as cases. Blood samples were taken to study IgM and IgG and saliva for genotype and RNA detection. The immunization status and the strain used were found through the vaccine distribution records. A univariate analysis was conducted on the data and relative risks calculated according to the vaccine strains used.

Results: A total of 63 cases were detected, 52 being students from one school. Fifty percent were $9-13$ years of age. A total of $88.5 \%$ of the cases detected at the school had been properly immunized. The IgM sensitivity was $9 \%$, PCR sensitivity being $37 \%$. The relative risk of those students immunized with an initial dose of Rubini strain as compared to those immunized with Jeryl-Lynn strain was 3.8 (95\% CI:2.27-6.49).

Conclusions: The high degree of immunization coverage does not prevent outbreaks from occurring in places having a high degree of exposure. The IgM reveals itself to be sensitive to a very small degree for the diagnosis of parotiditis. It seems necessary that the current immunization strategies and diagnostic methods be reconsidered.

Key words: Mumps. Mumps virus. Epidemiology. Vaccination. 


\section{INTRODUCCIÓN}

La parotiditis infecciosa es una enfermedad vírica aguda caracterizada por fiebre, hinchazón y dolor de una o más glándulas salivales, por lo regular la parótida. La complicación más frecuente es la orquitis, que suele ser unilateral y se presenta en el $20-30 \%$ de los hombres postpúberes; otras complicaciones son la pérdida de audición, ooforitis, pancreatitis, meningoencefalitis, miocarditis o artritis. Hasta un $50 \%$ de los casos puede ser asintomático o con síntomas respiratorios inespecíficos con o sin parotiditis ${ }^{1}$. Se transmite por vía aérea y por contacto directo con la saliva de una persona infectada. El período de incubación varía entre 14 y 25 días. El período de contagiosidad máxima transcurre desde unos dos días antes del comienzo de los síntomas hasta cuatro días después de que éstos aparezcan ${ }^{2}$. A partir de un caso índice el número de casos secundarios de infección esperados en población susceptible es de $10-12^{3}$.

La confirmación diagnóstica puede realizarse mediante pruebas serológicas (detección de IgM y elevación de IgG entre dos muestras), PCR para la detección de RNA en saliva y cultivo del virus ${ }^{4-7}$.

En Bizkaia la incidencia de parotiditis ha ido descendiendo paulatinamente en los últimos años, pasando de 3,42 casos por 100.000 habitantes en 1997 a 0,9 casos por 100.000 habitantes en 2005 , con una mayor afectación en los menores de 14 años (figura 1).

La vacuna del virus de la parotiditis se administra junto con las de sarampión y

Figura 1

Tasas de parotiditis vírica en Bizkaia (1997-2005)

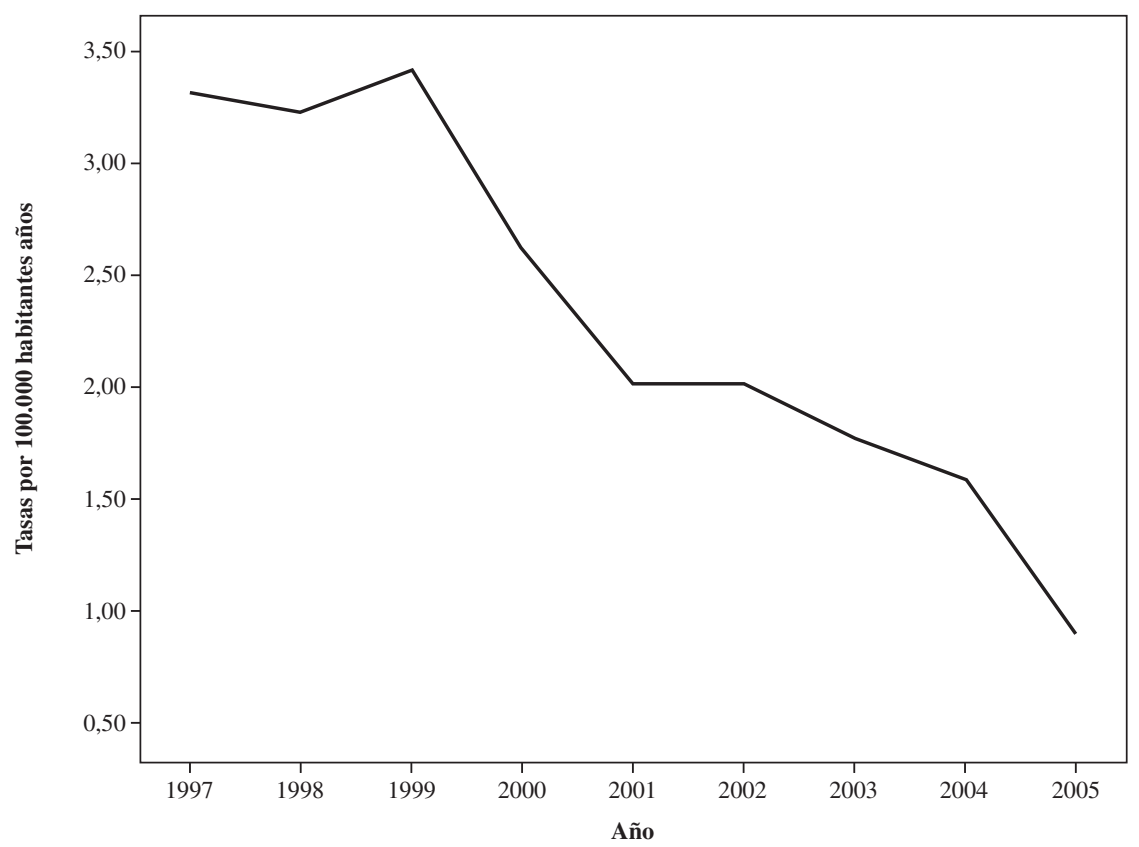

Fuente: EDO. 
rubéola en forma de triple vírica (TV). En el País Vasco se introdujo en el calendario oficial de vacunación en 1987 a la edad de 12 meses y desde 2000 se administra una segunda dosis a los 4 años de edad.

Diferentes estudios establecen una respuesta inmunitaria a la $1^{\mathrm{a}}$ dosis de TV en un $95 \%$ de los niños vacunados. Sin embargo, el $15 \%$ son seronegativos un año más tarde y el $25 \%$ entre 2 y 4 años más tarde. Con una segunda dosis se logra una mayor duración de niveles de anticuerpos ${ }^{8-}$ 11. La inmunización pasiva con inmunoglobulinas no parece ser útil en el control de brotes. La vacunación post-exposición no impide el desarrollo de la enfermedad, pues necesita entre 2 y 4 semanas para lograr completa inmunidad ${ }^{1,7,12}$. La cobertura vacunal necesaria para impedir la transmisión de la enfermedad se ha estimado en $90-92 \%{ }^{13}$.

En los últimos años se han dado importantes brotes no sólo en España sino también en Estados Unidos, Canadá y Reino Unido, entre otros países. Estos brotes afectaban principalmente a poblaciones susceptibles: personas no vacunadas o grupos de edad (de 15 a 23 años) que no habían tenido exposición al virus natural y tampoco estaban vacunados ${ }^{14-17}$. Sin embargo, ha habido también brotes en poblaciones con alta cobertura vacunal en las que se cuestionaba la efectividad de la cepa empleada para la vacunación, como en Suiza, Portugal, o España ${ }^{18-20}$. Los estudios de los brotes en estos países apuntaban hacia una escasa efectividad de la vacunación con el componente antiparotidítico de la cepa Rubini. En 1999 en la Comunidad Autónoma Vasca se decidió sustituir la cepa Rubini por la cepa JerrylLynn. En el año 2000 se modificó el calendario vacunal, adelantando la $2^{\mathrm{a}}$ dosis de vacuna TV de los 11 a los 4 años de edad y se realizó un catch-up a los niños y niñas entre 4 y 11 años. De esta manera se aseguraba que toda la pobla- ción de estas edades había recibido al menos una dosis de vacuna efectiva, en consonancia con las recomendaciones de la $\mathrm{OMS}^{21}$.

En marzo de 2006 se notificó a la Unidad de Vigilancia Epidemiológica una agrupación de casos de parotiditis que afectaba principalmente al alumnado del colegio de un municipio de Bizkaia y que dio lugar al mayor brote de parotiditis acontecido en los últimos años en este territorio.

El objetivo de este trabajo es describir y analizar dicho brote y ayudar a aclarar cuestiones en torno a la efectividad de las vacunas utilizadas en la actualidad o la sensibilidad de los métodos diagnósticos para la detección de parotiditis vírica, ya que sus resultados son la herramienta principal empleada a la hora de descartar esta enfermedad por los centros de vigilancia epidemiológica.

\section{MATERIAL Y MÉTODOS}

\section{Investigación epidemiológica}

Además del registro de los casos declarados en la forma habitual, se realizó una búsqueda activa a través del Sistema de las Enfermedades de Declaración Obligatoria (EDO) y del Sistema de Información Microbiológica (SIM) contactando telefónicamente con el personal de los centros de salud implicados para que declararan cualquier caso sospechoso. Se diseñó un registro de recogida de datos que incluía, además de los datos habituales de la declaración individual, las fechas de vacunación con TV y la cepa vacunal utilizada. En los casos necesarios los datos fueron completados mediante llamadas telefónicas al centro de salud.

Se establecieron definiciones de caso asociado al brote y de contacto susceptible (tabla 1), que, junto a las indicaciones per- 
Tabla 1

Definiciones establecidas de caso confirmado, probable y de contacto susceptible

\begin{tabular}{|l|l|}
\hline Caso confirmado por laboratorio & \multicolumn{1}{|c|}{ Definición } \\
\hline Caso confirmado por vínculo epidemiológico & $\begin{array}{l}\text { Hinchazón aguda unilateral o bilateral, sensible al tacto y autolimitada, de la parótida u otras } \\
\text { glándulas salivares cuya duración es superior a 2 días y sin que haya otras causas aparentes } \\
\text { Y Vínculo epidemiológico con el colegio y/o residente en el municipio Y Serología IgM(+), } \\
\text { aislamiento del virus en saliva o identificación de presencia del virus por técnicas de PCR en } \\
\text { saliva. }\end{array}$ \\
\hline Caso probable & $\begin{array}{l}\text { Hinchazón aguda unilateral o bilateral, sensible al tacto y autolimitada, de la parótida u otras } \\
\text { glándulas salivares cuya duración es superior a 2 días y sin que haya otras causas aparentes } \\
\text { Y Vínculo epidemiológico con el colegio y/o residente en el municipio. }\end{array}$ \\
\hline Contacto susceptible & $\begin{array}{l}\text { Hinchazón aguda unilateral o bilateral, sensible al tacto y autolimitada, de la parótida u otras } \\
\text { glándulas salivares cuya duración es superior a 2 días y sin que haya otras causas aparentes } \\
\text { Y Desconocemos (ni se confirma ni se descarta) la existencia de vínculo epidemiológico con } \\
\text { el colegio y/o residente en el municipio. }\end{array}$ \\
\hline & $\begin{array}{l}\text { Vínculo familiar, escolar, laboral, de amistad o de otro tipo relativamente cercano con un caso } \\
\text { de parotiditis vírica } \mathbf{Y} \text { No tiene antecedentes de parotiditis vírica Y Es menor de 41 años de } \\
\text { edad } \mathbf{Y} \text { Tiene entre 21 y 40 años y no tiene una dosis de TV O Tiene entre 4 y } 20 \text { años y no } \\
\text { tiene dos dosis de TV O Tiene entre 13 meses y 4 años de edad y sólo le han puesto una dosis } \\
\text { de TV. }\end{array}$ \\
\hline
\end{tabular}

tinentes, fueron difundidas entre el personal sanitario de los dos centros de salud del municipio, de cara a que estuvieran alerta ante nuevos casos y conocieran las pautas a aplicar: Los casos sospechosos fueron apartados del colegio o centro de trabajo durante el período estimado de transmisibilidad y se identificó a los contactos susceptibles. Se averiguó su estado vacunal y se completó la vacunación en caso necesario. El equipo encargado de la salud escolar en la zona revisó el estado vacunal de todo el alumnado para remitir a su centro de salud a los que tuvieran que completarlo.

Se informó del desarrollo del brote y de las medidas adoptadas a los centros de salud del municipio, así como a la Comarca Ezkerraldea-Enkarterri (Osakidetza) y a los Servicios Centrales de Salud Pública del Gobierno Vasco.

Para poder identificar el tipo de cepa vacunal que había recibido cada caso se averiguó qué lote correspondía ser administrado en la fecha de vacunación y el centro correspondientes en los registros de reparto de dosis vacunales de la Unidad de Vigilancia Epidemiológica de Bizkaia.

Para la estimación del riesgo relativo dentro del colegio en función de la cepa empleada para la vacunación se averiguó qué tipo de vacuna se había distribuido en el municipio en cada año y se calculó la probabilidad de haber recibido una vacuna Rubini en función del año de nacimiento.

Los datos poblacionales fueron extraídos del censo de 2001 del EUSTAT.

\section{Laboratorio}

Se recogieron muestras de suero para la determinación de $\operatorname{IgM}$ e $\mathrm{IgG}$, y de saliva para la realización de PCR.

Las pruebas serológicas se realizaron en el Laboratorio de microbiología del hospital de Cruces. Para la determinación de anticuerpos IgM frente al virus de la parotiditis se empleó el método ELISA (Enzyme-Linked lmmunosorbent Assay): Enzygnost AntiVirus de la parotiditis/IgM (Dade Behring, Germany). Para la determinación de anticuerpos $\operatorname{IgG}$ frente al virus de la parotiditis se utilizó el método ELFA (Enzyme Linked Fluorescent Assay): VIDAS Mumps IgG (Biomerieux, France) etiquetando como positivo un valor test (VT) mayor de 0,5.

Las muestras de saliva y orina fueron enviadas al Centro Nacional de Microbio- 
logía, del Instituto de Salud Carlos III, donde a su llegada al Laboratorio de Admisión de Muestras se realizaron dos alícuotas de $100 \mu \mathrm{l}$ que fueron conservadas a $-80^{\circ} \mathrm{C}$ hasta su procesamiento, el cual consistió en la extracción de ácidos nucleicos totales en una de las alícuotas mediante un extractor automático (MagNa Pure LC, ROCHE Diagnostics) siguiendo protocolo de lisis externa e incluyendo un plásmido de secuencia no relacionada con el virus de la parotiditis como parte del sistema de control interno. A continuación se buscó en los extractos ARN del virus de la parotiditis mediante una nested-RT-PCR dotada de un sistema de control interno para evitar falsos negativos $^{22}$. Todas aquellas muestras que no produjeron banda de control interna fueron ensayadas de nuevo partiendo de la segunda alícuota e informadas como inhibidas si en esta segunda amplificación tampoco se veía la banda. Todas las muestras con resultado positivo fueron confirmadas en una segunda nested-RTPCR para la amplificación de un fragmento del gen de la proteína SH y sólo fueron considerados los positivos en ambas $^{23}$. El genotipado de los virus detectados se hizo por secuenciación del mismo fragmento de amplificación del gen SH de la segunda RT-PCR ${ }^{23}$.

\section{RESULTADOS}

\section{Descripción epidemiológica}

Entre el 10 de febrero y el 13 de mayo de 2006 se detectaron 63 casos de parotiditis que respondían a la definición de caso confirmado. El primero fue un niño de 13 años del municipio de Portugalete y alumno del colegio implicado, correctamente vacunado con 2 dosis de TV y cuya analítica resultó IgM negativo.

El 92\% de los casos pertenecían al municipio de Portugalete y el $8 \%$ restante a poblaciones cercanas. El 75\% eran alumnos del colegio. De los 10 casos ajenos al colegio al menos 5 eran amigos o familiares de alumnos afectados.

El 50\% de los casos tenían entre 9 y 13 años, sin diferencias importantes en cuanto a la distribución por género.

Dentro de la sintomatología presentada la inflamación parotídea fue unilateral en el $70 \%$ y bilateral en el 30\% de los casos. Ninguno requirió ingreso hospitalario ni presentó complicaciones.

La tasa de ataque global dentro del municipio de Portugalete (51.066 habitantes, con un $33 \%$ de la población menor de 30 años) fue de 1,14 casos/1.000 habitantes (tabla 2).

El colegio implicado es un centro de estudios privado al que acuden 791 alumnos entre los 2 y los 18 años (40\% hombres y $60 \%$ mujeres) y 76 trabajadores. Está repartido en 2 edificios separados; en el primero se ubican los cursos hasta $2^{\circ}$ de ESO y en el segundo desde $3^{\circ}$ de ESO hasta $2^{\circ}$ de Bachiller. Los alumnos de ambos edificios comparten algunas instalaciones y profesorado.

Los 53 casos del colegio fueron 52 alumnos y una trabajadora. La tasa de ataque entre el alumnado fue de $6,57 \%(8,9 \%$ en niños y $5 \%$ en niñas) (tabla 3). El pico epidémico del brote se produjo en la semana 13 (26 de marzo a 1 de abril), con un total de 18 casos (figura 2).

Tabla 2

Brote de parotiditis. Tasas de ataque por grupos de edad. Portugalete 2006

\begin{tabular}{|c|c|c|}
\hline $\begin{array}{c}\text { Grupos de edad } \\
\text { (años) }\end{array}$ & Número de casos & $\begin{array}{l}\text { Tasa de ataque } \\
\left(n .^{\circ} \operatorname{casos} / 1.000\right)\end{array}$ \\
\hline $1-4$ & 3 & 2,24 \\
\hline $5-14$ & 45 & 12,81 \\
\hline $15-29$ & 6 & 0,54 \\
\hline$>29$ & 4 & 0,12 \\
\hline
\end{tabular}


Tabla 3

Brote de parotiditis. Tasas de ataque por curso Colegio. Portugalete 2006

\begin{tabular}{|c|c|c|}
\hline & $\mathbf{N}^{\mathbf{o}}$ casos & Tasa de ataque (\%) \\
\hline Edificio 1 & $\mathbf{4 8}$ & $\mathbf{8 , 2 2}$ \\
\hline $2^{\circ}$ infantil & 0 & 0 \\
\hline $3^{\circ}$ infantil & 1 & 1,96 \\
\hline $4^{\circ}$ infantil & 2 & 4 \\
\hline $5^{\circ}$ infantil & 0 & 0 \\
\hline $1^{\circ}$ primaria & 1 & 2,13 \\
\hline $2^{\circ}$ primaria & 2 & 4,44 \\
\hline $3^{\circ}$ primaria & 11 & 22,44 \\
\hline $4^{\circ}$ primaria & 7 & 13,7 \\
\hline $5^{\circ}$ primaria & 10 & 27,8 \\
\hline $6^{\circ}$ primaria & 6 & 9,23 \\
\hline $1^{\circ}$ ESO & 3 & 6 \\
\hline $2^{\circ}$ ESO & 5 & 7,5 \\
\hline Edificio 2 & $\mathbf{4}$ & $\mathbf{1 , 8 7}$ \\
\hline $3^{\circ}$ ESO & 2 & 3,3 \\
\hline $4^{\circ}$ ESO & 1 & 1,8 \\
\hline $1^{\circ}$ bachiller & 0 & 2,0 \\
\hline $2^{\circ}$ bachiller & & 0 \\
\hline
\end{tabular}

\section{Estado vacunal y eficacia de la vacuna} (tablas 4 y 5)

El 76\% de los casos había recibido dos dosis de vacuna TV y el $6 \%$ una sola dosis. Atendiendo al calendario de vacunación vigente y a su edad, el $88,5 \%$ de los casos dentro del colegio estaba correctamente vacunado. El estado vacunal era desconocido en el $17 \%$ de los casos.

El $40 \%$ de los vacunados había recibido cepa Rubini en su primera dosis de vacuna TV. La segunda dosis vacunal contenía la cepa Jeryl-Lynn en todos los casos.

Dentro del colegio se observó que los alumnos vacunados en su primera dosis con la cepa Rubini tenían casi cuatro veces más riesgo de enfermar de parotiditis que los vacunados con la cepa Jeryl-Lynn.

Figura 2

Brote de parotiditis. Curva epidémica. Portugalete 2006

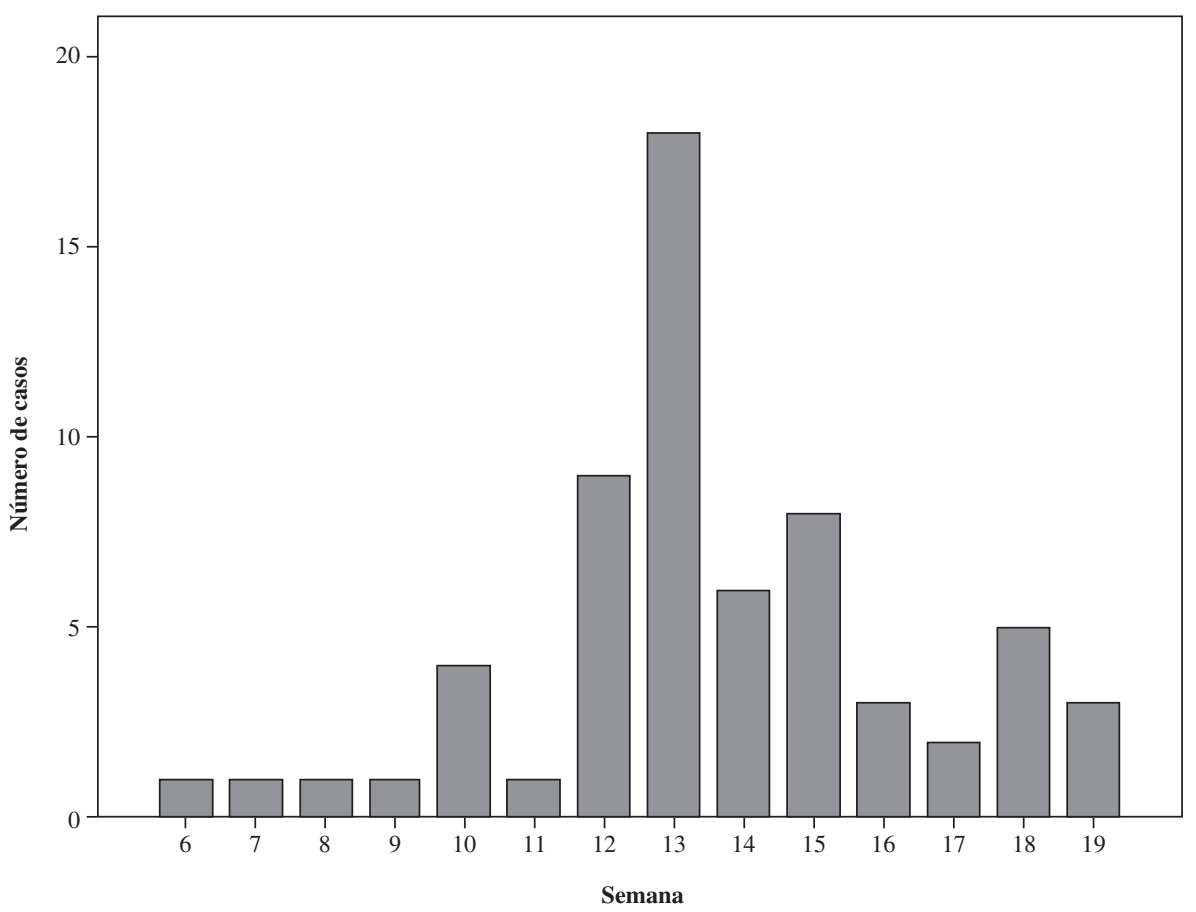


Tabla 4

Número de dosis de TV y tipo de cepa vacunal empleada según los grupos de edad

\begin{tabular}{|l|c|c|c|c|}
\hline & Vacunación no documentada & $\mathbf{1}$ dosis & $\mathbf{2}$ dosis & Total \\
\hline$<4$ años & 0 & $2(2 \mathrm{~J})$ & 0 & 2 \\
\hline $4-20$ años & 5 & $2(1 \mathrm{~J} / 1 \mathrm{R})$ & $48(28 \mathrm{~J} / 20 \mathrm{R})$ & 55 \\
\hline$>20$ años & 6 & 0 & 0 & $\mathbf{4 8}$ \\
\hline Total & $\mathbf{1 1}$ & $\mathbf{4}$ & $\mathbf{6 3}$ \\
\hline
\end{tabular}

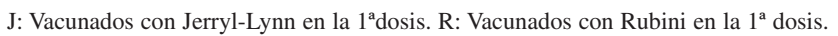

Tabla 5

Riesgo relativo de parotiditis dentro del alumnado en función la cepa vacunal empleada en la primera dosis

\begin{tabular}{|c|c|c|c|}
\hline \multirow{8}{*}{ 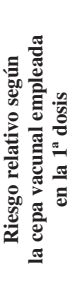 } & Todo el alumnado & \multicolumn{2}{|c|}{ RR 3,84 (IC95\% 2,27-6,49) } \\
\hline & Casos & Total alumnos & Cepa vacunal en la $1^{\text {a }}$ dosis \\
\hline & 19 & 104 & Rubini \\
\hline & 33 & 695 & Jeryl-Lynn \\
\hline & $\begin{array}{c}\text { Alumnos del edificio } 1 \text { con una } 2^{\mathrm{a}} \text { dosis } \\
\text { de TV administrada en } 2000\end{array}$ & \multicolumn{2}{|c|}{ RR 1,49 (IC95\% 0,71-3,13) } \\
\hline & Casos & Total alumnos & Cepa vacunal en la $1^{\text {a }}$ dosis \\
\hline & 8 & 51 & Rubini \\
\hline & 23 & 218 & Jeryl-Lynn \\
\hline
\end{tabular}

Cuando se seleccionaron únicamente las cohortes del edificio 1 , que recibieron una segunda dosis vacunal en el año 2000, se observó que los alumnos vacunados en su primera dosis con la cepa Rubini tenían 1,49 veces más riesgo de enfermar de parotiditis que los vacunados con la cepa Jeryl-Lynn.

\section{Resultados de laboratorio (tabla 6)}

Se obtuvieron muestras para serología de $\operatorname{IgM}$ en 58 de los 63 casos confirmados (92\%). La IgM fue positiva en el $9 \%$ de las muestras analizadas. Se realizó la determinación de IgG en 45 muestras y 43 de ellas fueron positivas. Se obtuvieron resultados para PCR en 27 muestras de saliva, de las que 10 fueron positivas.

Tabla 6

Resultados de laboratorio

\begin{tabular}{|l|c|c|c|c|}
\hline & Positivo & Negativo & Indeterminado & Total \\
\hline IgM & $5(9 \%)$ & $46(79 \%)$ & $7(12 \%)$ & 58 \\
\hline IgG & $43(96 \%)$ & $2(4 \%)$ & $0(0 \%)$ & 45 \\
\hline PCR & $10(37 \%)$ & $17(63 \%)$ & $0(0 \%)$ & 27 \\
\hline
\end{tabular}

El virus identificado en las muestras enviadas fue el virus de parotiditis genotipo G1.

\section{DISCUSIÓN}

A pesar de las altas coberturas vacunales con TV en la población infanto-juvenil de Bizkaia éste es el mayor brote de parotiditis declarado en este territorio en los últimos 10 años. El brote se dio principalmente en una población vacunada con al menos una dosis de cepa vacunal Jeryl-Lynn. Parece, sin embargo, que las tasas de ataque más elevadas se produjeron en las cohortes que habían sido vacunadas con la cepa Rubini en la primera dosis en su mayoría.

Los resultados obtenidos podrían conllevar cierto margen de error, ya que el método empleado para la estimación del tipo de cepa vacunal recibido por cada individuo se llevó a cabo a partir de las dosis distribuidas en las fechas y centros correspondientes; cabe la posibilidad de que en algún caso fuera otro lote el empleado por intercambio 
de vacunas entre centros, remanentes de lotes anteriores, vacunación en consultas privadas u otras circunstancias.

Teniendo en cuenta que la mayoría de los casos registrados habían recibido al menos una dosis de vacuna Jeryl-Lynn (y en muchos casos dos dosis) parece que la efectividad vacunal de esta cepa no es lo suficientemente alta como para evitar la aparición de brotes. Hasta la fecha la eficacia vacunal de la cepa Jeryl-Lynn se había estimado en un 60-80\% en situación de brote $^{24-26}$. Estos niveles se revelan insuficientes en lugares con un riesgo de exposición elevado y continuo, como los colegios, a pesar de alcanzarse altas coberturas vacunales.

No se encontraron diferencias en cuanto a la severidad de síntomas en función del estado vacunal y las cepas empleadas, pero el hecho de que la parotiditis pueda cursar hasta en un $20 \%$ de manera subclínica o hasta en un $50 \%$ con una presentación atípi$\mathrm{ca}^{1}$, podría haber ocasionado un infradiagnóstico de casos.

No se encontró asociación entre el tiempo transcurrido entre la última dosis vacunal y la tasa de ataque.

Llama la atención la baja sensibilidad de las pruebas diagnósticas empleadas. La determinación de IgM mediante ELISA sólo detectó el 8,3\% de los casos, coincidiendo con los resultados aportados por otros estudios ${ }^{27}$. El hecho de que se trate de una población vacunada en su mayoría, puede reducir el número de resultados IgM positivos, al no ser ésta la primera exposición al virus y haber podido desarrollar una respuesta inmune secundaria. Estos resultados apoyan la conveniencia de establecer pruebas diagnósticas más adecuadas para poblaciones con altas coberturas vacunales. Por otro lado, podrían haberse etiquetado como paperas otras enfermedades que hubieran cursado con clínica similar.
La determinación de la PCR en saliva también obtuvo una baja sensibilidad diagnóstica (37\%). La prueba mediante ELFA para determinar la IgG fue positiva en el $95,5 \%$ de los sujetos en los que se realizó, lo que a priori mostraría una alta sensibilidad del test. Sin embargo, el hecho de que no se haya realizado esta misma prueba en no enfermos, impide estimar su verdadera especificidad, ya que estos resultados podrían ser meros indicadores de seroconversión, independientemente de si ésta se ha producido por la enfermedad o por vacunación.

En la necesidad de buscar alternativas diagnósticas para la detección de parotiditis en poblaciones vacunadas sería planteable establecer nuevos puntos de corte en los niveles de $\operatorname{IgG}$ que ayudaran a identificar la infección reciente ${ }^{27}$. En este caso, al no disponer de IgG en la población no enferma, no se han podido establecer hipótesis a este respecto.

Según información aportada por el Centro Nacional de Microbiología, el genotipo G1 identificado en este brote, es el genotipo predominante en la actualidad a nivel nacional además de haber sido vinculado a otros brotes ocurridos recientemente en países como Estados Unidos, Reino Unido o Croacia, entre otros ${ }^{28,29}$. Por lo tanto, parece que nos hallamos ante una cepa que está circulando de una manera generalizada, no sólo en España sino también en Europa y Norteamérica.

Este brote, ocurrido principalmente en un centro escolar con una elevada cobertura vacunal, obliga a considerar una serie de aspectos:

- Ante una sintomatología compatible no descartar la posibilidad de parotiditis vírica, aún en el supuesto de individuos vacunados con 2 dosis. Un diagnóstico temprano facilitaría el control de los brotes. 
- Las pruebas diagnósticas utilizadas en la actualidad ante la sospecha de un caso de parotiditis deben replantearse ya que los test de detección de IgM han mostrado una baja sensibilidad en población vacunada.

- Ante la aparición de un nuevo caso debe realizarse vigilancia epidemiológica activa e investigación exhaustiva de los posibles brotes ${ }^{27}$.

- La vacuna antiparotidítica actualmente empleada no se revela tan efectiva como otras vacunas del calendario infantil (sarampión). Sin embargo, sí muestra un efecto protector si la comparamos con cepas menos inmunógenas (Rubini). Por lo tanto, es importante seguir manteniendo coberturas vacunales elevadas que ayuden a limitar la extensión de los brotes.

\section{AGRADECIMIENTOS}

A todo el equipo sanitario de la comarca Ezkerraldea-Enkarterri de Osakidetza y en especial a Esther Flores de Salud Escolar. A Victoria Martínez de Aragón del Centro Nacional de Epidemiología del Instituto de Salud Carlos III.

\section{BIBLIOGRAFÍA}

1. Plotkin SA. Mumps Vaccine. En: Plotkin SA, Orenstein WA, editores. Vaccines. Philadelphia: Elsevier;2003.p.441-69

2. Parotiditis infecciosa. En: Chin J editor. El control de las enfermedades transmisibles $17^{\mathrm{a}}$ ed. Washington: OPS;2001.p.499-502

3. Anderson RM, May RM. Modern vaccines: immunization and herd immunity. Lancet 1990;335:641-5

4. Gay N, Miller E, Hesketh P, Morgan-Capner P, Ramsay M, Cohen B et al. Mumps surveillance in England and Wales support introduction of two doses vaccination schedule. Commun Dis Rep Rev 1997;7:21-6
5. Perry KR, Brown DW, Parry JV, Panday S, Pipkin C, Richards A. Detection of measles, mumps and rubella antibodies using antibody capture immunoassay. J Med Virol 1993;40:235-40

6. Uchida K, Shinohara M, Shimada S, Segawa Y, Doi R, Gotoh A et al. Rapid and sensitive detection of mumps virus RNA directly from clinical samples by real-time PCR. J Med Vir 2005;75:470-4

7. Carvone KM, Wolinsky JS. Mumps virus. En: Knipe D, Howley P editores. Field virology. $4^{\mathrm{a}}$ ed. Philadelphia: Lippincot, Williams and Wilkins;2001.p.1381-1400

8. Vandermeulen C, Roelants M, Vermoere M, Roseew K, Goubau P, Hoppenbrouwers K. Outbreak of mumps in a vaccinated child population: a question of vaccine failure? Vaccine 2004;22:2713-6

9. Mitchell LA, Tingle AJ, Decarie D, Lajeunesse C. Serologic responses to measles, mumps and rubella (MMR) vaccine in healthy infants: failure to respond to measles and mumps components may influence decisions on timing of the second dose of MMR. Can J Public Health 1998;89:325-8

10. Pebody RG, Gay NJ, Hesketh LM, Vyse A, Morgan-Capner P, Brown D et al. Immunogenicity of second dose measles-mumps-rubella (MMR) vaccine and implications for serosurveillance. Vaccine 2002;20:1134-40

11. Davidkin I, Valle M, Julkunen I. Persistence of anti-mumps virus antibodies after a two-dose MMR vaccination. A nine year follow-up. Vaccine 1995;13:1617-22

12. Centers for Disease Control and Prevention. Measles, mumps, and rubella-vaccine use and strategies for measles, rubella, and congenital rubella syndrome elimination and mumps control: Recommendations of the Advisory Committee on Immunization Practices (ACIP). MMWR Morb Mortal Wkly Rep 1998; 47 (RR-8): 1-57

13. Anderson R, May R. Immunization and herd immunity. Lancet 1990;335:641-5

14. Gupta RK, Best J, Mac Mahon E. Mumps and the UK epidemic 2005. BMJ 2005;330:1132-5

15. Visser LE, Gonzáles Pérez LC, Ramos Tejera J, Berjón Barrientos AC, Vergara Guerrero Y, Martines navarro F. An outbreak of mumps in the province of Leon Spain 1995-1996. Euro Surveill 1998;3:14-8

16. Public Health Agency of Canada. Outbreak of mumps among young adults-Vancouver, British 
Columbia.[citado 15 de nov. 1997]. http://www.phacaspc.gc.ca/publicat/ccdr-rmtc/97vol23/dr2322ea.html

17. Centers for Disease Control and Prevention. Update: Multistate outbreak of mumps-United States, January 1-May 2,2006.MMWR Morb Mortal Wkly Rep 2006;55:1-5

18. Chamot E, Toscani L, Egger P, German D, Bourquin C. Estimation of the efficacy of the strains of mumps vaccines during an epidemic of mumps in the Geneva Canton (Switzerland). Rev Epidemiol Sante Publique 1998;46:100-7

19. Limón Mora J, Molina Lopez T, Domínguez Camacho JC. Community effectiveness of vaccines against infectious parotiditis (mumps). Report of cases. Rev Esp Salud Pública 1999;73:455-64

20. Gonçalves G, De Araújo A, Monteiro Cardoso ML. Outbreak of mumps associated with poor vaccine efficacy-Oporto Portugal 1996. Euro Surveill 1998;3:119-21

21. Word Health Organization. Mumps virus vaccines. Wkly Epidemiol Rec. 2001;76:345-56

22. Poggio GP, Rodríguez C, Cisterna D, Freire MC, Cello J. Nested PCR for rapid detection of mumps virus in cerebrospinal fluid from patients with neurological diseases. J Clin Microbiol 2000;38(1):274-278

23. Palacios G, Jabado O, Cisterna D, de Ory F, Renwick N, Echevarria JE et al. Molecular identifica- tion of mumps virus genotypes from clinical simples: Standarized method of analysis. J Clin Microbiol 2005;43(4):1869-1878

24. Richard JL, Zwahlen M, Feuz M, Matter HC. Comparison of the effectiveness of two mumps vaccines during an outbreak in Switzerland in 1999 and 20000: a case-cohort study. Eur J Epidemiol 2003;18:569-77

25. Harling R, White JM, Ramsay ME, Macsween KF, Van den Bosch C. The effectiveness of the mumps component of the MMR vaccine: a case control study. Vaccine 2004;23:4070-74

26. Vandermeulen C, Roelants M, Vermoere M, Roseeuw K, Goubau P, Hoppenbrouers K. Outbreak of mumps in a vaccinated child population: a question of vaccine failure?. Vaccine 2004;22:2713-6

27. De los Ríos Martín R, García Marín N, Sanz Moreno JC, Ballester Orcal E. Parotiditis en un área urbana de la Comunidad de Madrid. Estado vacunal, diagnóstico y medidas de intervención Aten Primaria 2001;28:10-16

28. Centers of Disease Control and Prevention. Mumps epidemic-Iowa,2006.MMWR Morb Mortal Wkly Rep 2006;55:366-68

29. S̆antak M, Ko\%outiç-Gulija T, Te\%ooviç G, Ljubin-Sternak S, Gjenero-Margan I, Betica-Radiç L, Forãiç D. Mumps virus strains isolated in Croatia in 1998 and 2005: Genotyping and putative antigenic relatedness to vaccine strains. J Med Virol. 2006;78:638-43 